\title{
Driving magnetic activity: differential rotation, flow structures, and surface patterns
}

\author{
Klaus G. Strassmeier \\ Leibniz-Institute for Astrophysics Potsdam (AIP), An der Sternwarte 16, D-14482 Potsdam, \\ Germany \\ email: kstrassmeier@aip.de
}

\begin{abstract}
The interplay between stellar rotation and turbulent flows is a major ingredient for vertical angular momentum transport in stellar convection zone. Combined with the centrifugal force and the buoyancy force due to pole-equator temperature gradients one can expect a largescale flow structure that is usually referred to as differential rotation and meridional flows. I review such observations for stars other than the Sun, mostly for stars significantly more active, and ask the question whether such observations can constrain the dynamo process.
\end{abstract}

Keywords. Stars: activity, Stars: rotation, Stars: spots, Sun: rotation, Magnetic fields

\section{The Sun as a role model}

The solar differential rotation is the result of the interplay between angular momentum transport by the Reynolds stress of rotating convection, a large-scale meridional flow, and a slight anisotropy in the convective heat transport. Nowadays, the Reynolds stress theory of the solar differential rotation reproduces the solar rotation law as known from helioseismology in great detail (Kitchatinov \& Rüdiger 2005). In order to compute the differential rotation of a solar-type star the stratification of pressure, density and temperature are needed as input parameters. Küker \& Stix (2001) computed first differential rotation models for the Sun for rotation periods between 56 days to 7 days. The stratifications were taken from a solar model but did not yet include the complex surface layers. These and subsequent computations (e.g. Küker et al. 2011) suggest an increased horizontal shear for a 56-day rotating Sun by $\approx 70 \%$ more than for today's Sun. The meridional flow shows one cell per hemisphere and points toward the equator on the surface and toward the pole at the bottom of the convection zone. A maximum meridional flow velocity of $9.2 \mathrm{~m} \mathrm{~s}^{-1}$ at the surface is predicted. For a 28-day rotating Sun the model of the meridional flow pattern consist of two cells per hemisphere, with the flow directed toward the equator at both the bottom and the top of the convection zone and poleward at intermediate depths. For a 14-day rotating Sun the normalized horizontal shear further decreases to $\approx 50 \%$ of today's value. There are still two flow cells per hemisphere, but the outer cell is restricted to a very shallow surface layer. The surface meridional flow is polewards with a flow speed of $3.8 \mathrm{~m} \mathrm{~s}^{-1}$. Further decreasing the rotation period to seven days leads to a further reduction of the shear to less than a factor four with respect to today's Sun. Unfortunately, no stellar detections of meridional flows on stars other than the Sun exist to date. 


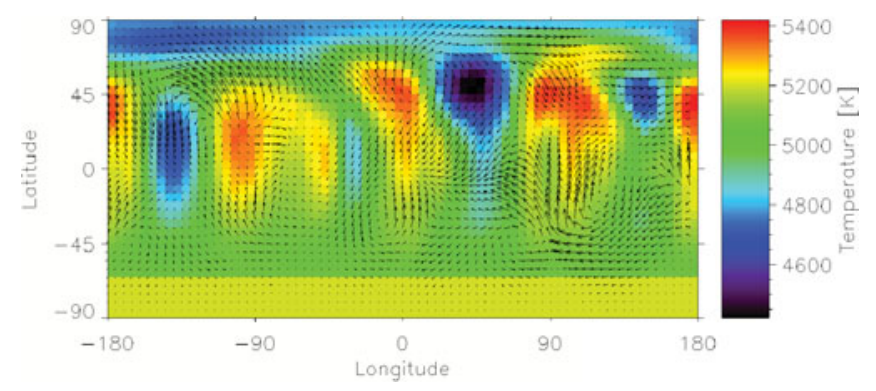

Figure 1. Surface velocity map (arrows) of the K2 dwarf LQ Hydrae from local-correlation tracking. The background color/shading is the average temperature map from the full epoch of Doppler imaging.

\section{Differential rotation and meridional flows on the Sun and other stars}

We typically simplify the "solar-type" differential rotation law by

$$
\Omega(b)=\Omega_{\mathrm{eq}}-d \Omega \sin ^{2} b,
$$

where $b$ is the surface latitude, $\Omega(b)$ the respective rotation rate at a particular latitude, $\Omega_{\mathrm{eq}}$ the rotation rate at the equator, and $d \Omega$ the difference in rotation rate between the pole and the equator. The paper by Wöhl et al. (2010) gives an enlightening summary of the various solar differential rotation laws derived from a large range of tracers. Their closest representation of the observational data was obtained with

$$
\Omega_{\odot}(b)=14.499 \pm 0.006-2.54 \pm 0.06 \sin ^{2} b-0.77 \pm 0.09 \sin ^{4} b .
$$

$\Omega(b)$ represents the sidereal rotation velocity in degree per day.

I also present the current version of the ZDI code iMap in which the sheared image analysis is applied to temperature structures for the WTTS V410 Tau (Carroll et al. 2012). Only a very weak solar-like differential rotation is present, if at all. The formal value for the angular velocity at the equator is $\Omega_{e q}=3.356 \pm 0.005$, well within the errors of the photometrically determined rotation period, and for the differential rotation rate between the equator and the pole $d \Omega=0.007 \pm 0.009$. We conclude that differential rotation of this very active single WTTS must be very small, likely smaller than a factor 30 than on the Sun. For another very active star, the K0 giant XX Tri, we found a shear of 0.023 with a corresponding lap time of $1050 \mathrm{~d}$, thus moderately strong differential rotation for a $10.7-\mathrm{R}_{\odot}$ star. We also present the results of an analysis of the very active K2 dwarf LQ Hya (Flores-Soriano et al. 2012) where we found a shear of 0.01 and a lap time of $150 \mathrm{~d}$. The time series Doppler-imaging data even allowed the application of a solar-like, local-correlation tracking as shown in Fig. 1. Meridional flows were also computed for a variety of stars by Küker et al. (2011). Observations like in Fig. 1 would principally allow to search for such flows but no conclusive evidence is present and likely better data are required.

\section{The link to the dynamo}

A dynamo mechanism comparable to the Sun's converts mechanical energy into magnetic flux and transports it up to the surface where it is observable as spots, plages, flares, etc.. It has been proposed that the morphology of the emerging magnetic flux, i.e. flux tubes, allows a glimpse back into the star (e.g. Schüssler et al. 1996). Despite that 


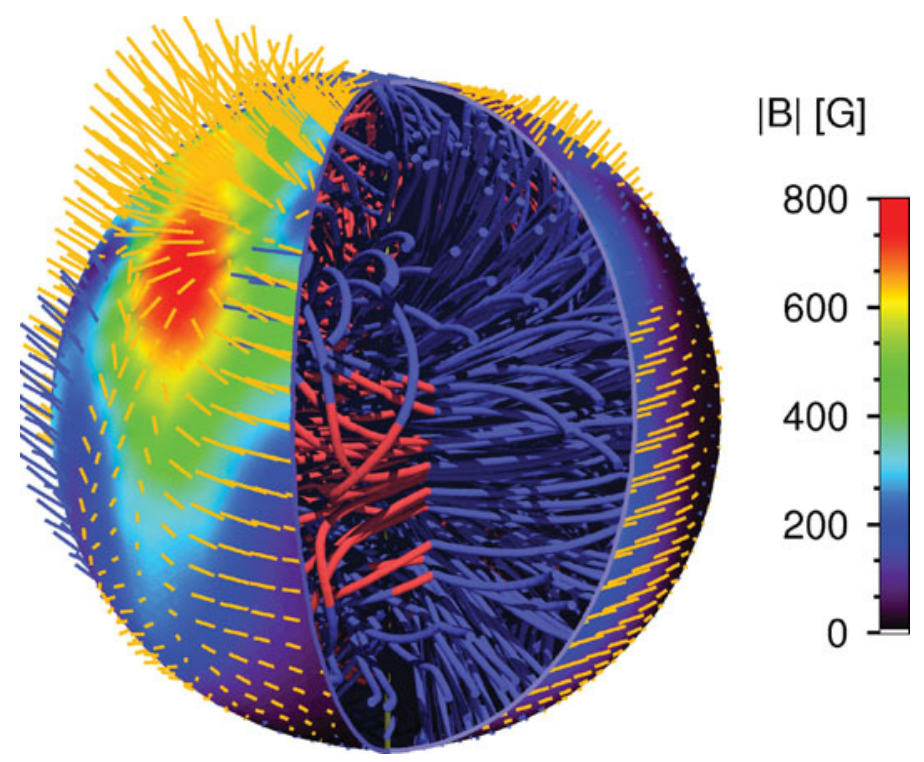

Figure 2. A combination of a mean-field dynamo simulation with a Zeeman-Doppler image of II Pegasi, a K2 subgiant. The simulation and the ZDI were obtained completely independent but the figure shows the potential of using ZDI images as the outer boundary condition for global numerical dynamo simulations.

many folks do not "believe" in flux tubes in the first place, they are an excellent modeling agent for (magnetic) flux and momentum transport to the surface. We demonstrate this visually in Fig. 2 for the active subgiant II Peg. There is mounting evidence that even the quiet solar photosphere is not field-free but rather permeated by small-scale, mixed polarity magnetic fields that show no significant variation with the solar activity cycle (see e.g. Trujillo Bueno et al. 2004). It has been suggested that a considerable part of the quiet Sun magnetic fields may be produced by a self-excited turbulent dynamo operating in the near-surface layers of the Sun, even though the flow in these layers is not noticeably affected by rotation and thus exhibits no net helicity (Cattaneo 1999, and subsequent papers). In principle, near-surface magneto-convection simulations could provide a selfconsistent description of a local dynamo, including a prediction of its contribution to the mean magnetic flux of the quiet Sun and other stars.

\section{Acknowledgements}

I would like to thank the organizers for the invitation to speak.

\section{References}

Carroll, T. A., Strassmeier, K. G., Rice, J. B., \& Künstler, A. 2012, A\&\&A, in press

Cattaneo, F. 1999, ApJ 515, L39

Flores-Soriano, M., Strassmeier, K. G., \& Küker, M. 2012, Poster paper Cool Stars 17, Barcelona

Kitchatinov, L. L. \& Rüdiger, G. 2005, AN 326, 379

Küker, M. \& Stix, M. 2001, A\&A 366, 668

Küker, M., Rüdiger, G., \& Kitchatinov, L. L. 2011, A\&A 530, A48

Schüssler, M., Caligari, P., Ferriz-Mas, A., Solanki, S. K., \& Stix, M. 1996, A\&\&A 314, 503

Trujillo Bueno, J., Shchukina, N., \& Asensio Ramos, A. 2004, Nature 430, 326

Wöhl, H., Brajsa, R., Hanslmeier, A., \& Gissot, S. F. 2010, A\&A 520, A29 